\title{
MGMT Gene Promoter Methylation
}

National Cancer Institute

\section{Source}

National Cancer Institute. MGMT Gene Promoter Methylation. NCI Thesaurus. Code C153562.

A genetic finding indicating that DNA methylation in the promoter region of the MGMT gene has been detected in a sample. 